\title{
A Specific Assessment of the Normal Anatomy of the Aortic Root in Relation to Age and Gender
}

\author{
Xiang Wang ${ }^{1,2}$ \\ Xin-Shuang Ren $^{3}$ \\ Yun-Qiang $\mathrm{An}^{3}$ \\ Zhi-Hui Hou ${ }^{3}$ \\ Yi-Tong $\mathrm{Yu}^{3}$ \\ Bin $\mathrm{Lu}^{3, *}$ \\ Fang Wang ${ }^{1,2, *}$ \\ 'Department of Cardiology, Beijing \\ Hospital, National Center of \\ Gerontology, Institute of Geriatric \\ Medicine, Chinese Academy of Medical \\ Sciences, Beijing, 100730, People's \\ Republic of China; ${ }^{2}$ Graduate School of \\ Peking Union Medical College, Chinese \\ Academy of Medical Science, Beijing, \\ 100730, People's Republic of China; \\ ${ }^{3}$ Department of Radiology, Fuwai \\ Hospital, National Center for \\ Cardiovascular Diseases, Chinese \\ Academy of Medical Sciences and Peking \\ Union Medical College, Beijing, I00037, \\ People's Republic of China \\ *These authors contributed equally to \\ this work
}

Correspondence: Fang Wang Department of Cardiology, Beijing Hospital, National Center of Gerontology, Institute of Geriatric Medicine, Chinese Academy of Medical Sciences, No. I DaHua Road, Dongcheng District, Beijing, 100730, People's

Republic of China

Tel +86 I0 85132266

Email fw_wangdr@163.com

Bin Lu

Department of Radiology, Fuwai Hospital, National Center for Cardiovascular

Diseases, Chinese Academy of Medical

Sciences and Peking Union Medical

College, No. 167 Bei-Li-Shi Street,

Xicheng District, Beijing, 100037, People's

Republic of China

Tel +861088398700

Email binlu_bl09@I63.com
Background: A limitation associated with coronary computed tomography angiography (CCTA) is the lack of a normal reference value for aortic root dimensions and the uncertainty of the influence of age and gender on these dimensions. The purpose of the present study was to identify the normal values and variations of aortic root dimensions in healthy individuals and investigate how gender and age affect aortic root size.

Methods: A total of 1286 healthy yellow population $(52.7 \pm 11.0$ years, 634 male) who underwent CCTA were retrospectively included in the present study. Male and female patients were divided into seven groups according to age ( $<30$ years old, 30-39, 40-49, $50-59,60-69,70-79, \geq 80$ years old). In these age groups, we measured and compared the parameters of the aortic root.

Results: After body surface area (BSA) correction, the aortic root parameters of females were found to be greater than those of males in the 40-49 age group $(\mathrm{P}<0.05)$. There were no significant differences in aortic root parameters between genders in other age groups, except for the diameter of the ascending aorta, which was greater in females $(\mathrm{P}<0.05)$. In males, age was positively correlated with aortic root parameters $(\mathrm{P}<0.05)$, except for the annulus short diameter and LVOT short diameter. In females, age was positively correlated with aortic root parameters $(\mathrm{P}<0.05)$, except for the left coronary ostia height and the LVOT short diameter. Conclusion: Aortic root dimensions are affected by age and gender. After BSA correction, females show larger aortic root dimensions than males, and aortic root diameters increase with age.

Keywords: aortic root, BSA, CCTA, age, gender

\section{Introduction}

Accurate assessment of the aortic root anatomy is crucial for the detection of aortic root disease and for the preoperative selection of an appropriate size valve. Accordingly, pre-operative imaging plays an important role in assessing the anatomy of the aortic root. ${ }^{1}$ Data on the aortic root have been collected from transesophageal echocardiography, coronary computed tomography angiography (CCTA), and magnetic resonance imaging. ${ }^{1-3}$ CCTA has the advantage of providing threedimensional images of the aortic root anatomy, as well as the relationship of the annulus to the coronary ostia, with a high spatial resolution. CT is considered a promising non-invasive imaging modality for aortic root morphology assessment and diagnosis of aortic root disease, ${ }^{4-7}$ showing superiority over other modalities. ${ }^{8}$ However, many of the routinely used CT imaging planes do not have a normal reference value for aortic root dimensions, and little is known about the influence of age and gender on the dimensions of the aortic root. 
The purpose of the present study was to identify the normal values of aortic root dimensions from a large population examined by CCTA. The effect of gender and age on aortic root dimension differences was also explored.

\section{Methods}

\section{Patients}

Participants were recruited from the Fuwai Hospital, National Center for Cardiovascular Diseases, a single center study on the yellow population. We retrospectively analyzed 3018 patients suspected of coronary artery disease without aortic and aortic valve disease who underwent CCTA from June 2016 to March 2017. All the patients were initially recommended for CCTA by their cardiologists. The study complied with the Declaration of Helsinki, and all patients provided informed consent for inclusion in the registry. Subjects with heart valve disease $(n=192)$, heart failure $(n=130)$, congenital heart disease $(\mathrm{n}=97)$, cardiomyopathy $(\mathrm{n}=106)$, left ventricular hypertrophy $(\mathrm{n}=77)$, atrial fibrillation $(\mathrm{n}=195)$, or hypertension $(n=808)$ were excluded from the study. Subjects who did not have the complete clinical information necessary to calculate the pre-test $(n=117)$ non-diagnostic coronary images due to motion artifacts or inadequate image contrast on CCTA scans $(n=10)$ were also excluded. In total, 1286 patients were included in the study. The literature provides little basis for determining "normal" aortic root morphology; therefore, patients without any pathologic findings that could affect the aortic root anatomy were selected. This retrospective study was approved by the Ethics committee of Fuwai Hospital, National Center for Cardiovascular Diseases, Chinese Academy of Medical Sciences and Peking Union Medical College.

\section{CCTA Protocol}

All patients were examined with a second-generation dualsource CT system (SOMATOM Definition Flash; Siemens Healthcare, Forchheim, Germany). A $100 \mathrm{kV}$ tube voltage was used for patients with a body mass index (BMI) $<30 \mathrm{~kg} / \mathrm{m}^{2}$, while a $120 \mathrm{kV}$ tube voltage was used for patients with a BMI $\geq 30 \mathrm{~kg} / \mathrm{m}^{2}$. The $\mathrm{x}$-ray tube current was adjusted individually for each patient according to BMI. Automated bolus tracking was used in regions of interest within the ascending aorta for contrast medium enhancement, with a signal attenuation trigger threshold of 100 Hounsfield units (HU) and a six-second scan delay.
The main scanning parameters were kept at $0.6 \mathrm{~mm}$ individual detector width, $280 \mathrm{~ms}$ gantry rotation time, 0.20 0.50 pitch, and a $200-250 \mathrm{~mm}$ field of view for raw image reconstruction. A triple-phase contrast medium injection protocol that consisted of 50 to $60 \mathrm{~mL}$ undiluted contrast agent (Iopromide, $370 \mathrm{mgI} / \mathrm{mL}$; Bayer Healthcare, Berlin, Germany) was used and was followed by a $30 \mathrm{~mL} \mathrm{30:70}$ mixture of contrast medium and saline and a $30 \mathrm{~mL}$ saline chaser bolus, all injected at flow rates of 4 to $5 \mathrm{~mL} / \mathrm{s}$. All images were acquired during systole (usually in the 20 $50 \%$ phase of the cardiac cycle), as slightly larger annular sizes have been noted in the systolic phase than in the diastolic phase. ${ }^{2,9-12}$

\section{Clinical Data}

A structured review of existing clinical data was conducted after the CCTA to collect information on symptoms, demographic characteristics, and the presence of cardiovascular risk factors. Hypercholesterolemia was defined by the current use of lipid-lowering medication or by National Cholesterol Education Panel (NCEP) guidelines. ${ }^{12}$ Diabetes mellitus was defined by a previously established diagnosis, insulin therapy, or oral hypoglycemic therapy. Obesity was defined by a body mass index $>30$. The body surface area (BSA) was calculated using the Stevenson's formula BSA $\left(\mathrm{m}^{2}\right)=$ $0.0061 \times$ Height $(\mathrm{cm})+0.0128 \times$ Weight $(\mathrm{kg})-0.1529$ for the Chinese patient population. ${ }^{13}$ Previous studies have shown a significant correlation between aortic root diameter and BSA and have suggested that correction of aortic dimensions for BSA is a valid, simplified approach for uniformly correcting aortic dimensions for body size at all aortic sites. ${ }^{13,14}$ Therefore, to eliminate the influence of body size, each parameter was calibrated using BSA to better standardize male and female aortic dimensions. Moreover, the parameters of the aortic root diameter/ BSA were used to better explore the relationship between aortic root dimensions, gender, and age in the present study. Seven subgroups in each sex based on age were defined: $<30$ years, 30 to 39 years, 40 to 49 years, 50 to 59 years, 60 to 69 years, 70 to 79 years, and $\geq 80$ years.

\section{CCTA Image Analysis}

Based on the need for transcatheter aortic valve implantation (TAVI), parameters were chosen, including the dimensions of the aortic annulus, sinus of Valsalva (SOV), sinotubular junction (STJ), left ventricular outflow tract (LVOT), and ascending aorta. The aortic annulus was 
defined as an oval structure virtually identifiable at the level of the basal attachment of aortic cusps. ${ }^{10}$ All image post-processing and analyses were conducted on a commercially available medical workstation (Deep Blue, ADW4.6, GE Healthcare, Milwaukee, WI, US). Data were loaded into a post-processing package of Cardiac Reformat, and images were reconstructed in the coronal, sagittal, and axial orientation. The images were subsequently analyzed using a multiplanar oblique tool by two experienced radiologists. The aortic annulus was gained by double oblique transverse reconstruction of the aortic root below the three lowest insertion points of the aortic cusps. An oblique transversal plane perpendicular to the aortic annulus plane was used to measure the length of the LVOT, the width of the aortic sinus, and the width of the STJ (Figure 1). The coronary ostia height was measured on the oblique coronal or sagittal plane (Figure 2). Post-processing methods and measurement procedures for assessing the aortic root are described in detail in prior related literature..$^{2,11,12}$

Following this, the aortic root parameters were measured using multiplanar reformations according to expert consensus guidelines: (1) aortic annulus plan: to measure the short and long diameter and the mean diameter (the mean diameter $=($ long diameter + short diameter $) / 2) ;(2)$ coronary ostia height: the distance from the coronary ostia to the aortic annulus plane; (3) LVOT plan (5 $\mathrm{mm}$ below the aortic annulus): to measure the short and long diameter; (4) width of the SOV; (5) width of the STJ; ${ }^{11,15}$ and (6) diameter of the ascending aorta $(40 \mathrm{~mm}$ above the aortic annulus). ${ }^{10}$

\section{Statistical Analysis}

All statistical analyses were conducted using commercially available software (SPSS 24 for Windows, SPSS Inc, Chicago, IL, USA). The continuous variables were

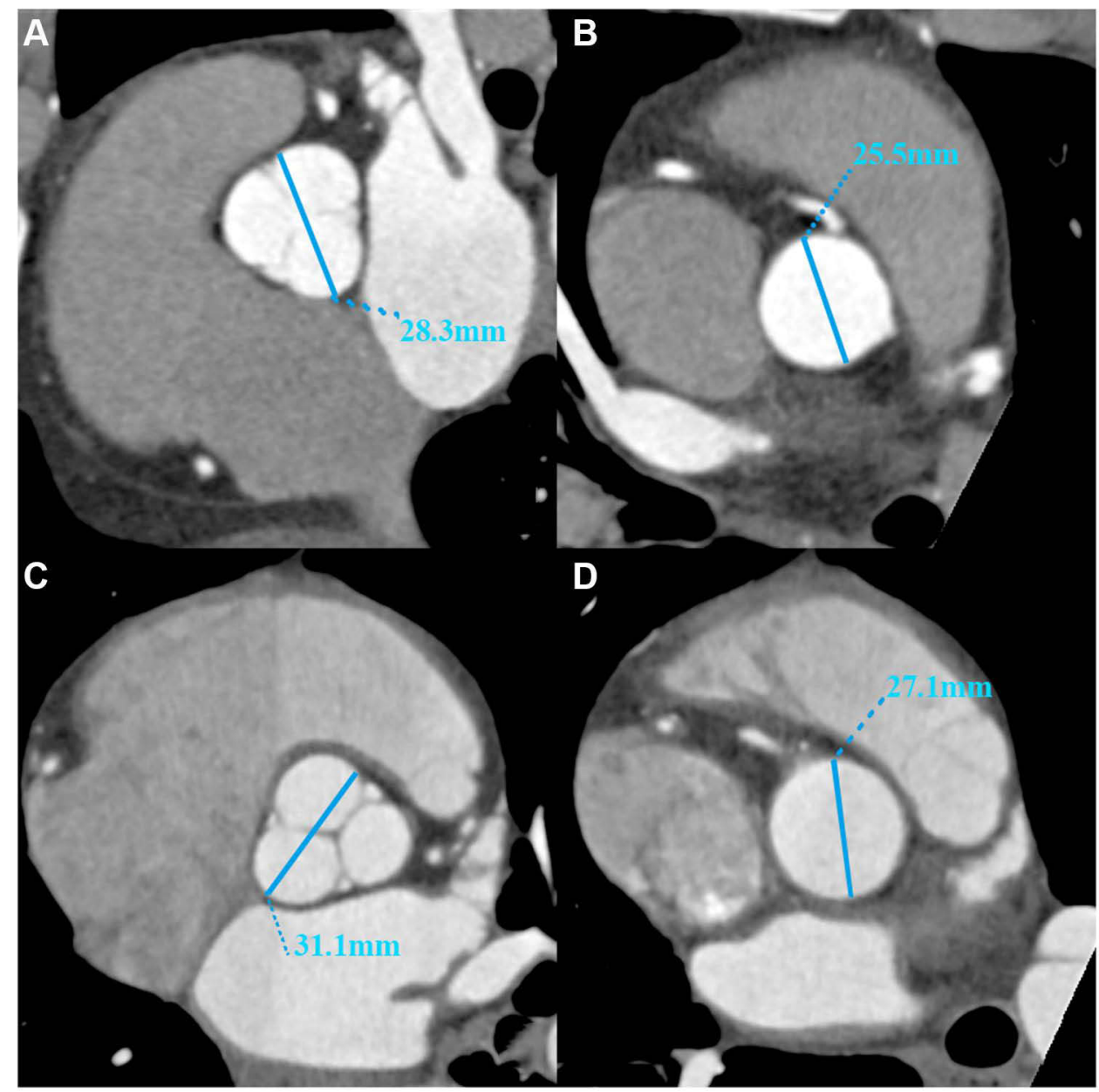

Figure I Computed tomography angiogram oblique transversal plane perpendicular to the aortic annulus plane in a 25 -year-old male (A): width of the SOV; (B) width of the STJ) and a 59-year-old male (C) width of the SOV (D) width of the STJ. 


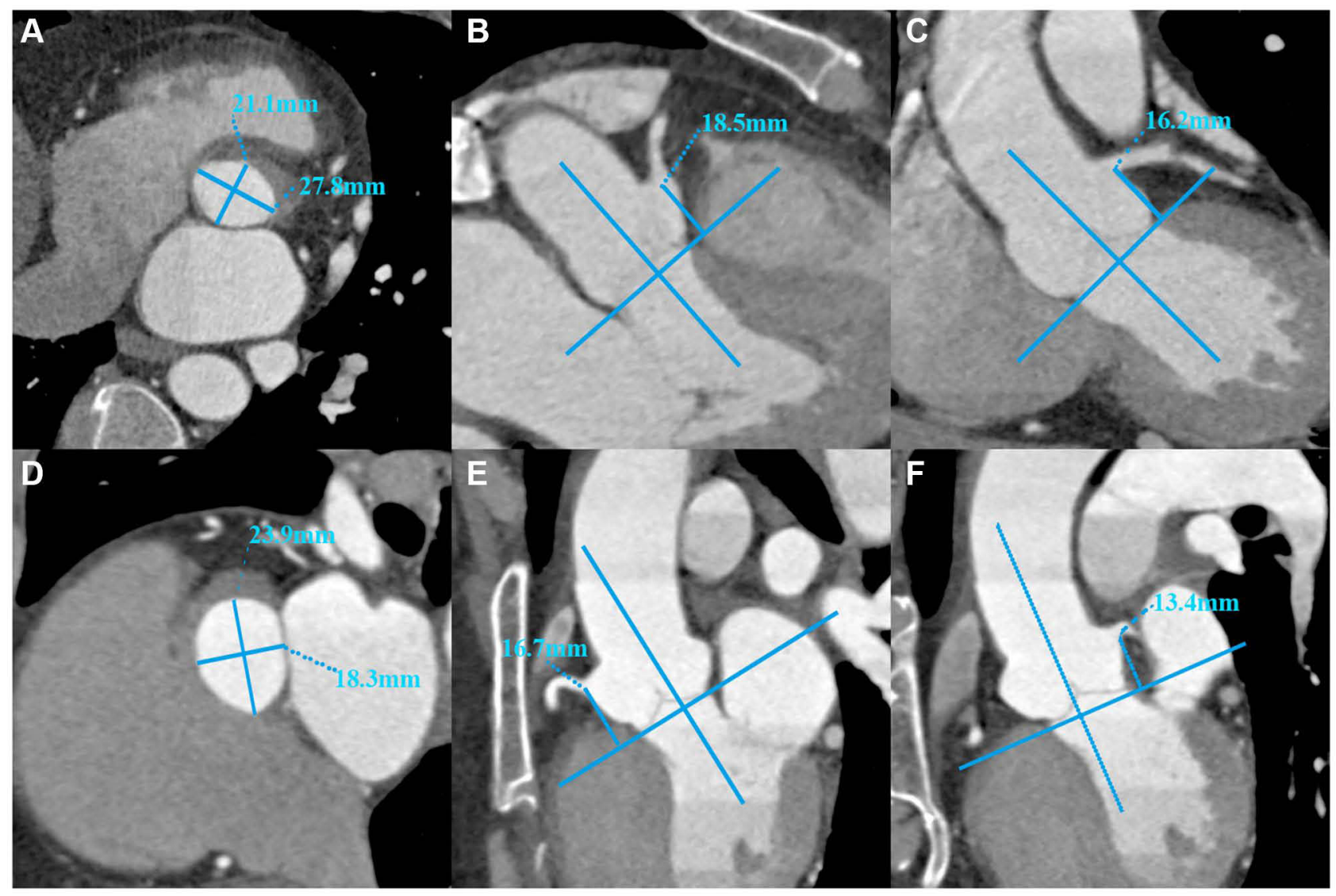

Figure 2 Computed tomography angiographic double oblique transverse images of the aortic annulus and coronary ostia height in a 53-year-old male (A) Aortic annulus dimension; (B) Right ostia to annulus; (C) Left coronary ostia to annulus) and 54-year-old female (D) Aortic annulus dimension; (E) Right ostia to annulus; (F) Left coronary ostia to annulus.

expressed as mean value \pm standard deviation after the assessment of normal distribution. A one-way ANOVA was used to compare the continuous variables in different age groups according to the normality of distribution and homogeneity of variance. An unpaired Student's $t$-test was used to compare the continuous variables between men and women in each age group. A Pearson correlation was used to evaluate the association between age and aortic root dimensions. A two-tailed p-value of less than 0.05 was considered statistically significant.

\section{Results}

\section{Subject Demographics}

Of the 1286 patients, the mean age was $52.7 \pm 11.0$ years old (ranging from 18 to 95 years), with an age distribution of $<30$ years $(1.63 \%), 30-39$ years $(9.72 \%), 40-49$ years (28.69\%), 50-59 years (31.96\%), 60-69 years $(22.40 \%)$, $70-79$ years $(4.43 \%)$, and $\geq 80$ years $(1.17 \%)$.

Table 1 shows the patients' baseline characteristics. Tables 2 and 3 show male and female BSA-corrected parameters of aortic roots in different age groups. Table 4 shows the correlation between BSA-corrected aortic root parameters and age. Figures 3 and 4 showed the trends in male and female aortic root parameters as affected by age, both before and after BSA correction, respectively.

\section{Aortic Annulus Dimensions}

In each age group, the male subjects' aortic annulus dimensions were significantly higher than those of the

Table I Patients' Baseline Characteristics

\begin{tabular}{|l|c|c|}
\hline Variable & Male & Female \\
\hline Number & 634 & 652 \\
Age (years) & $50.8 \pm 11.1$ & $54.6 \pm 10.7$ \\
BSA & $1.87 \pm 0.15$ & $1.61 \pm 0.12$ \\
Obesity & $56(8.8 \%)$ & $22(3.4 \%)$ \\
Diabetes & $47(7.4 \%)$ & $36(5.5 \%)$ \\
Dyslipidemia & $119(18.8 \%)$ & $124(19.0 \%)$ \\
\hline
\end{tabular}

Abbreviation: BSA: body surface area $\left(\mathrm{m}^{2}\right)$ 

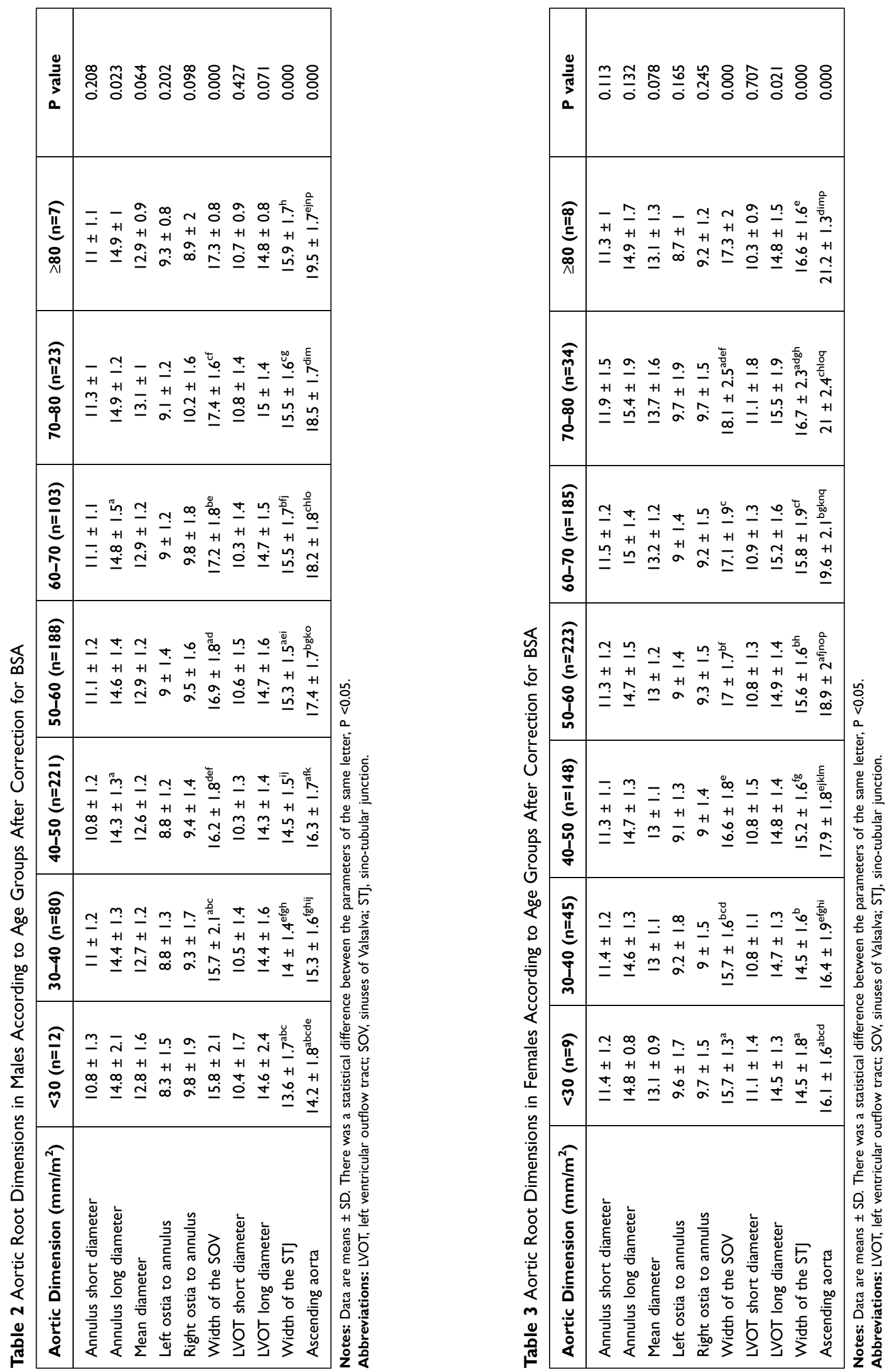
Table 4 Correlation of Aortic Root Dimension with Age After Correction for BSA

\begin{tabular}{|l|c|c|c|c|}
\hline \multirow{2}{*}{} & \multicolumn{2}{|c|}{ Male } & \multicolumn{2}{c|}{ Female } \\
\cline { 2 - 5 } & $\mathbf{r}$ & P value & $\mathbf{r}$ & P value \\
\hline Annulus short diameter & 0.058 & 0.142 & 0.082 & 0.037 \\
Annulus long diameter & 0.126 & 0.001 & 0.094 & 0.016 \\
Mean diameter & 0.101 & 0.011 & 0.096 & 0.015 \\
Left ostia to annulus & 0.099 & 0.012 & -0.009 & 0.814 \\
Right ostia to annulus & 0.097 & 0.015 & 0.066 & 0.095 \\
Width of the SOV & 0.272 & 0.000 & 0.242 & 0.000 \\
LVOT short diameter & 0.032 & 0.426 & 0.025 & 0.528 \\
LVOT long diameter & 0.103 & 0.009 & 0.138 & 0.000 \\
Width of the STJ & 0.326 & 0.000 & 0.258 & 0.000 \\
Ascending aorta & 0.533 & 0.000 & 0.494 & 0.000 \\
\hline
\end{tabular}

Abbreviations: LVOT, left ventricular outflow tract; SOV, sinuses of Valsalva; STJ, sino-tubular junction.

female subjects $(\mathrm{P}<0.05$; Figure $3 \mathrm{~A}-\mathrm{C})$. However, the female subjects' aortic annulus dimensions were higher than those of the male subjects after BSA correction. The difference was significant in the 40-49 age groups (short diameter: male $10.8 \pm 1.2 \mathrm{~mm}$ vs female $11.3 \pm 1.1 \mathrm{~mm}$; long diameter: male $14.3 \pm 1.3 \mathrm{~mm}$ vs female $14.7 \pm$ $1.3 \mathrm{~mm}$; mean diameter: male $12.6 \pm 1.2 \mathrm{~mm}$ vs female $13 \pm 1.1 \mathrm{~mm}$; all $\mathrm{P}<0.001$ ) and $60-69$ age groups (short diameter: male $11.1 \pm 1.1 \mathrm{~mm}$ vs female $11.5 \pm 1.2 \mathrm{~mm}$; long diameter: male $14.8 \pm 1.5 \mathrm{~mm}$ vs female $15 \pm$ $1.4 \mathrm{~mm}$; mean diameter: male $12.9 \pm 1.2 \mathrm{~mm}$ vs female $13.2 \pm 1.2 \mathrm{~mm}$; all $\mathrm{P}<0.001$ ), whereas the other five age groups showed no statistical differences (Figure 4A-C). Statistical differences were found in the annulus short diameter and the mean diameter between different age groups $(\mathrm{P}<0.05)$ for male subjects, though only the annulus long diameter was statistically different after BSA correction ( $\mathrm{P}=0.023$; Table 2 ). Age was positively correlated with the annulus long diameter and mean diameter in males ( $\mathrm{r}=0.126, \mathrm{r}=0.101$, respectively; $\mathrm{P}<0.05$; Table 4 ). In females, there was no statistical difference in the aortic annulus dimensions in all age groups before and after BSA correction (Table 3), whereas age was positively correlated with aortic annulus dimensions $(r=0.08, r=0.094$, and $\mathrm{r}=0.096$, respectively; $\mathrm{P}<0.05$; Table 4).

\section{LVOT, SOV, STJ, and Ascending Aorta Dimensions}

In male subjects, all parameters were higher than those of females in each age group, though there was no difference in the LVOT short diameter in patients $<30$ years old, the diameter of the ascending aorta in patients $<30$ years old and $>70$ years old, and the width of the STJ in patients $\geq$ 80 years old (all $\mathrm{P}>0.05$; Figure $3 \mathrm{~F}-\mathrm{J}$ ). However, after correcting for BSA, females' LVOT diameters were higher than those of males in patients 40-49 years old (short diameter: male $13.3 \pm 1.3 \mathrm{~mm}$ vs female $10.8 \pm 1.5 \mathrm{~mm}$, $\mathrm{P}=0.002$; long diameter: male $14.3 \pm 1.4 \mathrm{~mm}$ vs female $14.8 \pm 1.4 \mathrm{~mm}, \mathrm{P}<0.001$ ) and $60-79$ years old (short diameter: male $10.3 \pm 1.4 \mathrm{~mm}$ vs female $10.9 \pm 1.3 \mathrm{~mm}$, $\mathrm{P}<0.001$; long diameter: male $14.7 \pm 1.5 \mathrm{~mm}$ vs female $15.2 \pm 1.6 \mathrm{~mm}, \mathrm{P}=0.004)$; the SOV dimensions for females were higher than those of males in patients $40-49$ years old (male $16.2 \pm 1.8 \mathrm{~mm}$ vs female $16.6 \pm 1.8 \mathrm{~mm}$, $\mathrm{P}=0.015$ ); the STJ dimensions for females were higher than those of males in patients 30-39 years old (male 14 $\pm 1.4 \mathrm{~mm}$ vs female $14.5 \pm 1.6 \mathrm{~mm}, \mathrm{P}=0.04)$ and $40-49$ years old (male $14 \pm 1.4 \mathrm{~mm}$ vs female $15.2 \pm 1.6 \mathrm{~mm}$, $\mathrm{P}<0.001)$; the ascending aorta dimensions for females were higher than those of males at all ages $(\mathrm{P}<0.05$, all; Figure 4F-J). In male subjects, the other aortic root values were statistically different among different age groups $(\mathrm{P}<0.05)$, except for the LVOT long diameter. However, after BSA correction, the SOV, STJ, and ascending aorta dimensions were statistically different among all age groups $(\mathrm{P}<0.001$; Table 2$)$. In female subjects, statistical differences were detected in the width of the SOV and ascending aorta (all $\mathrm{P}<0.05$ ). However, after BSA correction, the LVOT long diameter, SOV, STJ, and ascending aorta dimensions showed statistical differences among age groups $(\mathrm{P}<0.001$; Table 3$)$. After BSA correction, age was shown to be positively correlated with the LVOT long diameter, the width of the SOV, the STJ, and the ascending aorta, regardless of gender (male: $\mathrm{r}=0.103, \mathrm{r}=0.272$, $\mathrm{r}=0.326, \mathrm{r}=0.533$, respectively; $\mathrm{P}<0.005$; female: $\mathrm{r}=0.138$, $\mathrm{r}=0.242, \mathrm{r}=0.258, \mathrm{r}=0.494$, respectively; $\mathrm{P}<0.001$ ) but was correlated with the LVOT short diameter (Table 4).

\section{Right and Left Coronary Ostia Height}

The left coronary ostia height in males exceeded that of females in the following age groups: $30-40$ years $(\mathrm{P}<0.001), 40-50$ years $(\mathrm{P}<0.001), 50-60$ years $(\mathrm{P}<0.001)$, 60-70 years $(\mathrm{P}=0.003)$ and $\geq 80$ years $(\mathrm{P}=0.004$; Figure $3 \mathrm{E})$. The right coronary ostia height in males exceeded that of females in the following age groups: < 30 years $(\mathrm{P}=0.022), 30-40$ years $(\mathrm{P}<0.001), 40-50$ years $(\mathrm{P}<0.001), 50-60$ years $(\mathrm{P}<0.001), 60-70$ years $(\mathrm{P}<0.001)$ and $70-80$ years $(\mathrm{P}<0.001$; Figure $3 \mathrm{D})$. However, after BSA correction, the left coronary ostia height was higher in 
- male

A

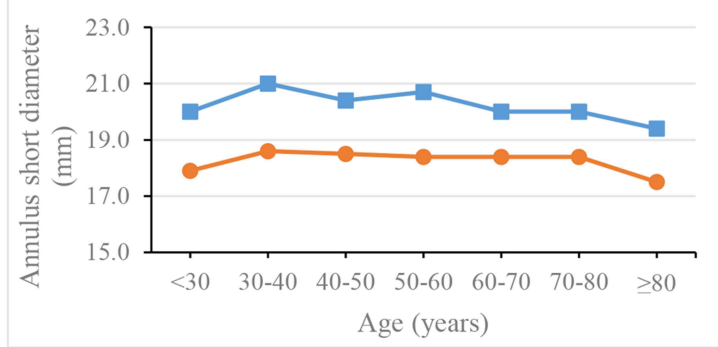

\section{C}

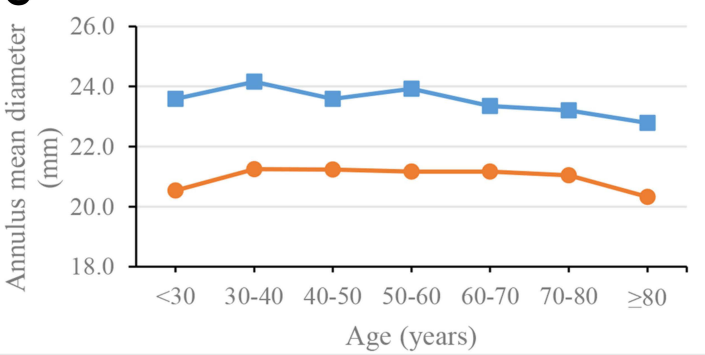

E

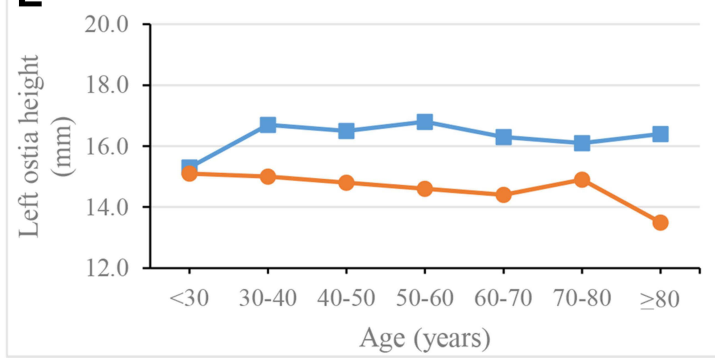

G

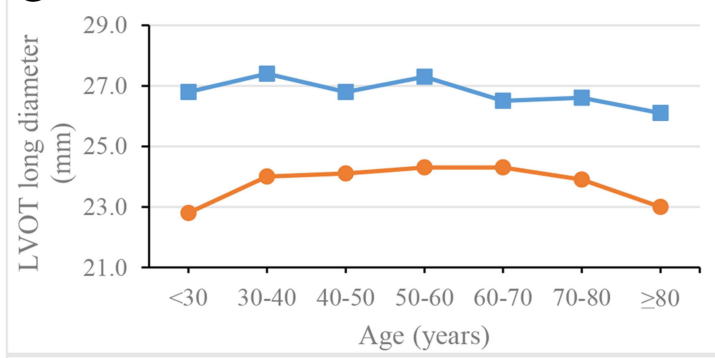

I

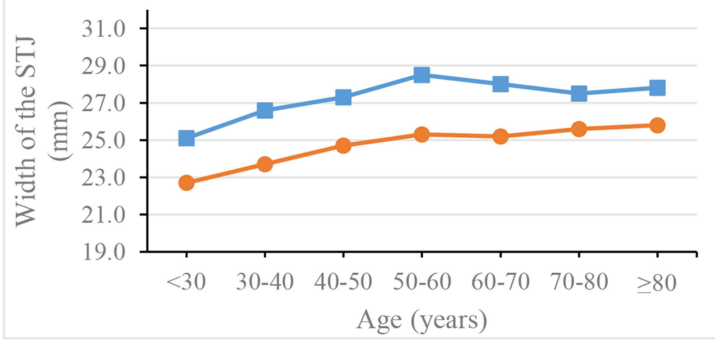

female

B

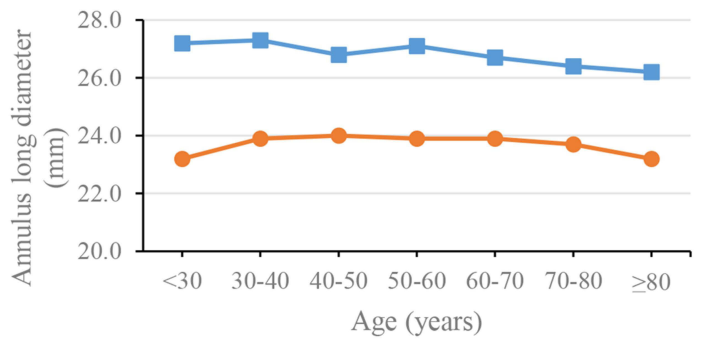

D
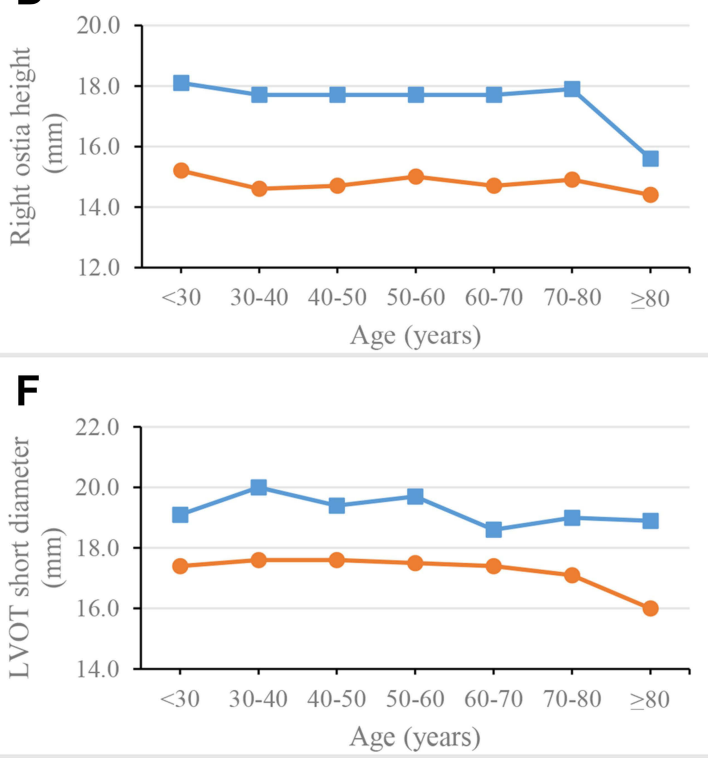

H

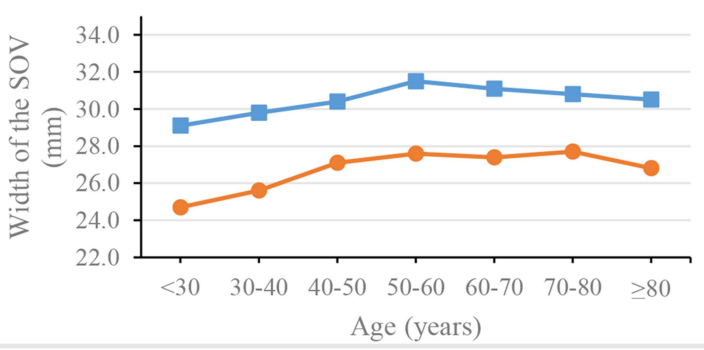

J

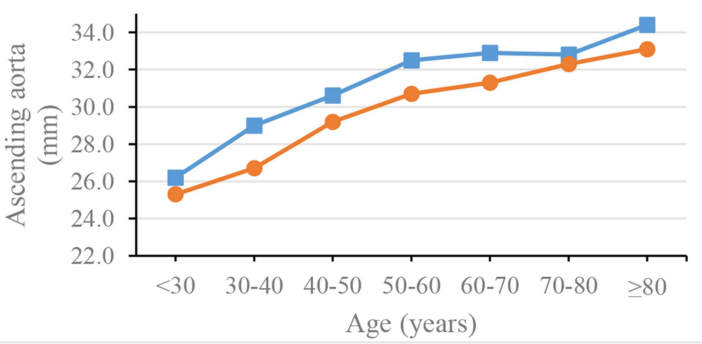

Figure 3 Trend of aortic root parameter values with age. (A) Annulus short diameter; (B) Annulus long diameter; (C) Annulus mean diameter; (D) Right ostia to annulus; (E) Left ostia to annulus; (F) LVOT short diameter; (G) LVOT long diameter; (H) Width of the SOV; (I) Width of the STJ; (J) Ascending aorta.

Abbreviations: LVOT, left ventricular outflow tract; SOV, sinuses of Valsalva; STJ, sino-tubular junction. 
- male

A

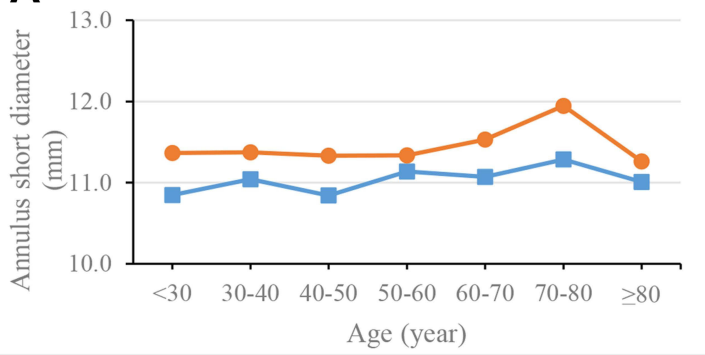

C
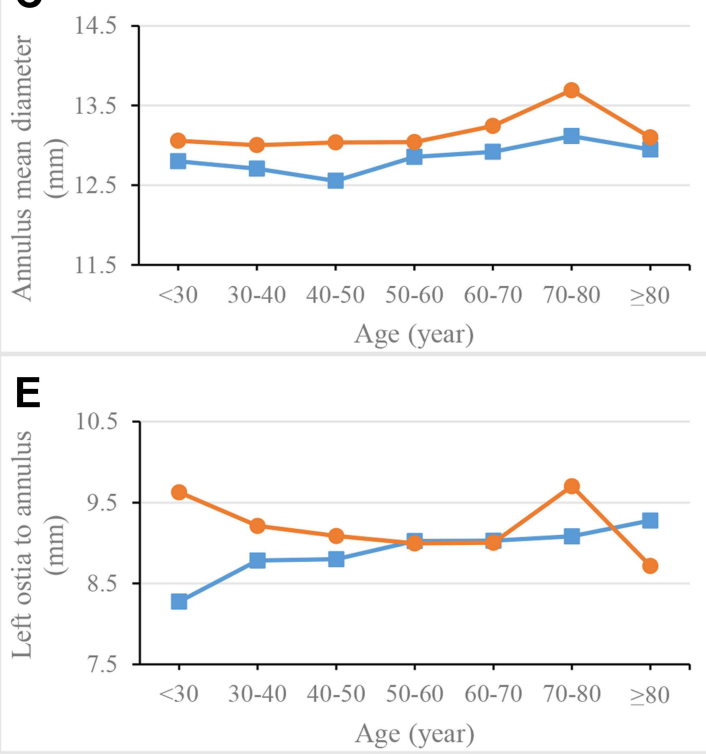

G
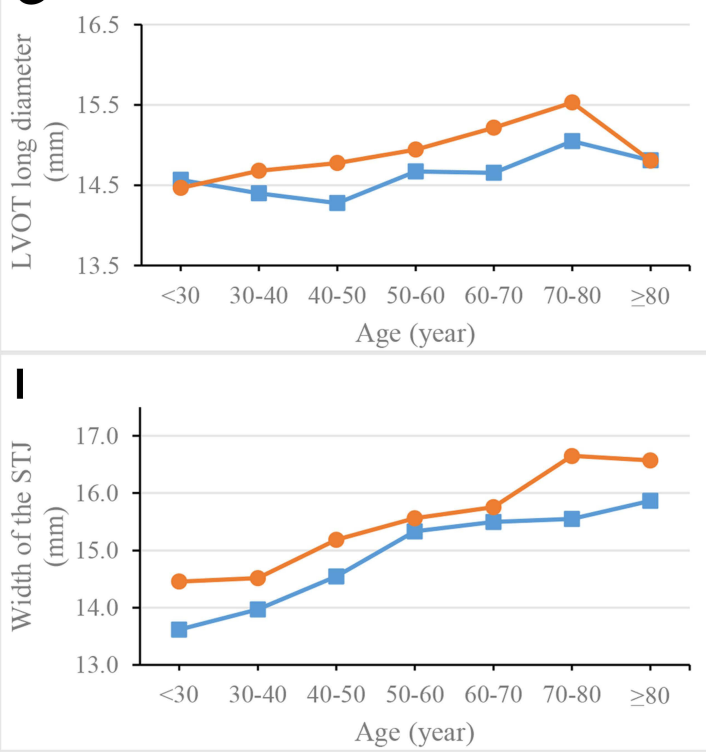

B

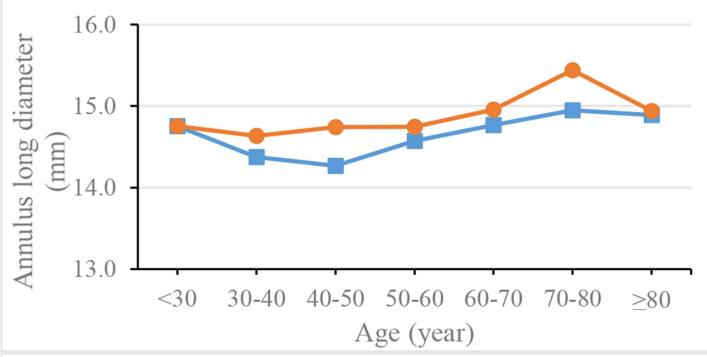

D

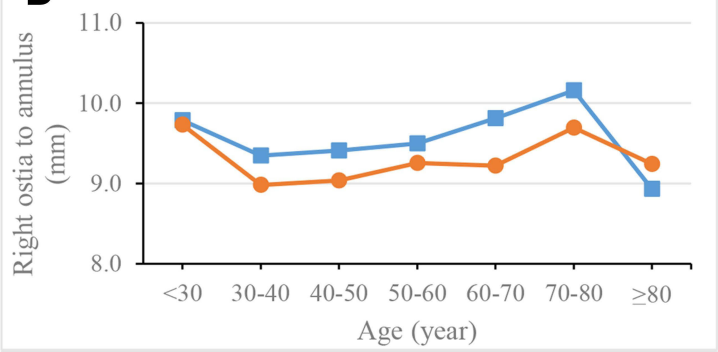

F

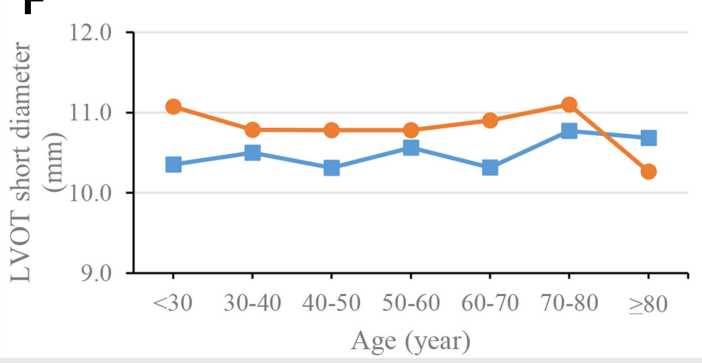

H

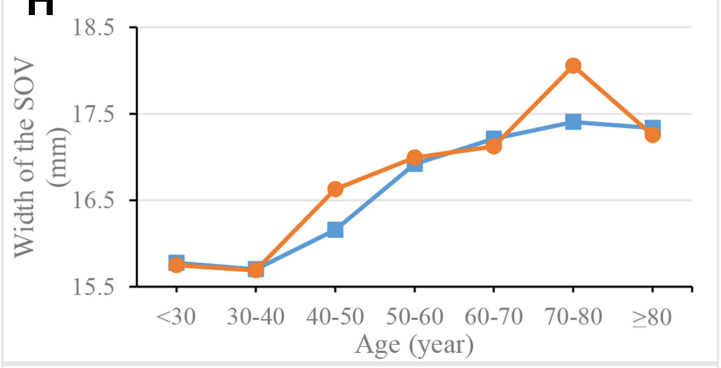

J

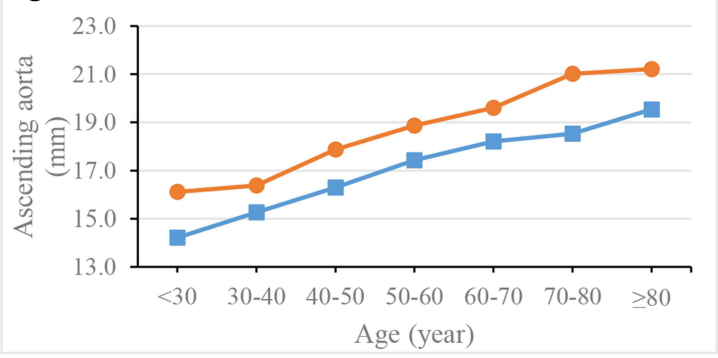

Figure 4 Trend of aortic root parameter values with age after BSA correction. (A) Annulus short diameter; (B) Annulus long diameter; (C) Annulus mean diameter; (D) Right ostia to annulus; (E) Left ostia to annulus; (F) LVOT short diameter; (G) LVOT long diameter; (H) Width of the SOV; (I) Width of the STJ; (J) Ascending aorta. Abbreviations: LVOT, left ventricular outflow tract; SOV, sinuses of Valsalva; STJ, sino-tubular junction. 
females than in males, and statistical significance was detected in the 40-50 year age group $(\mathrm{P}=0.039$; Figure 4E). The right coronary ostia height for females was higher than that of males in patients 40-50 years old and $60-70$ years old $(\mathrm{P}=0.009, \mathrm{P}=0.003$, respectively; Figure 4D). There was no statistical difference in the value of the coronary ostia height among age groups (Table 2). In addition, females showed no correlation regarding coronary ostia height, while males showed a positive correlation between age and coronary ostia height (left: $\mathrm{r}=0.099, \mathrm{P}=0.012$; right: $\mathrm{r}=0.097, \mathrm{P}=0.015$ ).

\section{Discussion}

Accurately measuring the aortic root size is greatly important in diagnosing aortic root disease (aortic dissection, stenosis, or aneurysm formation) and in preoperative guidance for TAVI surgery. The present study assessed the relationship between aortic root dimensions determined by CCTA and age plus gender in a large Chinese population of relatively normal individuals $\geq 18$ years old. The aortic annulus diameter in the Chinese population was found to be similar to that of a Western population reported in previous research. ${ }^{16,17}$ In contrast, the dimensions of the ascending aorta and the width of STJ in the Western population were higher than those in the Chinese population. Ethnic differences, including height and weight, may be responsible for the different aortic root dimensions observed. The present method of aortic root evaluation may also have had an impact on the different dimensions observed because CT was used in the present study, whereas previous studies used transesophageal echocardiography and magnetic resonance imaging.

The relationship between age and thoracic aortic dimensions has been reported many times in prior literature, ${ }^{14,18}$ including in research that suggested that the aortic annulus is not affected by increasing age. ${ }^{17}$ However, aging only partially explains changes in the aortic size in patient populations. The observed dilatation incensement could potentially be caused by age-related collagen synthesis. ${ }^{19}$ However, as the findings of the present study suggest, after BSA correction, age may have a varying effect on different aortic root segments, such as a stronger relationship to the dimensions of the diameter of the ascending aorta. In addition, consistent with other published data, the ascending aortic diameter, the width of the SOV, and the STJ diameter showed good correlation with age. ${ }^{17,21,22}$ Most previous studies have utilized transesophageal echocardiography and magnetic resonance imaging on a small patient population, whereas the present study employed CT on a large patient population with a broad age distribution. The present large patient population, wide range of ages, CT imaging method, and BSA correction method could account for the differences in aortic root dimensions to those measured in prior studies.

Regarding the relationship between aortic root dimensions and gender, previous research has shown that males have a larger aorta size and have also found an association with larger body size. ${ }^{16}$ In one study on 700 healthy Japanese individuals ages 20-79 years, echocardiogram revealed that the width of the SOV was $3 \mathrm{~mm}$ larger in men than women; however, multivariate analyses adjusting for body size were not reported. ${ }^{21}$ In the present study, most of the aortic root parameters showed significant gender differences. Before BSA correction, males' aortic root dimensions were higher compared with those of females; however, after BSA correction, females' aortic root dimensions were higher than those in males. Similar findings were reported by Arik Wolak et al, who showed that women had wider inner diameters of the ascending aorta than men following BSA correction. ${ }^{16}$ In the present study, most of the aortic root parameters showed significant gender differences in the 40-50 year age group only, except for the diameter of the ascending aorta. This could be due to the large number of patients included in this age group or could also be because previous studies used transesophageal echocardiography to conduct the measurements and did not employ BSA correction. In the present study, the aortic root parameter values were found to increase with age until age $\geq 80$ years, at which point the values suddenly decreased. In addition, the width of the STJ and the ascending aortic dimensions were found to have significant gender differences with increasing age. On the one hand, aortic root morphology is often subject to pathologic changes, such as connective tissue disease, bicuspid aortic valve, ${ }^{23}$ aortic stenosis, and atherosclerosis; therefore, studying the normal aortic root is beneficial in detecting these diseases. On the other hand, differences in aortic parameters exist between different age and gender groups; therefore, individualized assessment can improve the accuracy of aortic root disease diagnosis. The races studied by Pham et al were different from the present study. ${ }^{24}$ They mainly measured Caucasian people, with inconsistent measurement parameters and a small age range mainly around 50 years old, without age grouping. In the yellow population we measured, the aortic root parameters were more detailed, the sample size was relatively large, and the age group was more detailed. 
Similarly, it was found that SOV/STJ/ascending aorta was correlated with age. It provides detailed data support for the diagnosis and treatment of main artery root diseases and valvular diseases. For example, in TAVI surgery, accurate preoperative aortic root parameters can avoid implant valve falling off, perivalvular leakage, aortic dissection or rupture, and coronary artery mouth obstruction, thus improving the success rate of surgery.

The present study has some limitations. First, the sample size of certain age groups was relatively small, particularly individuals $<30$ years old and $\geq 70$ years old. Second, the present study evaluated patients from a single center in China, which may limit the application of the results in different ethnicities. Lastly, patients enrolled in the present study were suspected of coronary artery disease but did not have aortic and aortic valve disease.

\section{Conclusion}

In summary, significant differences in aortic root dimensions were found to be affected by age and gender. CCTA showed that males had larger aortic root dimensions than females, and the Sinus, SOV, and ascending aorta increased with age. However, after BSA correction, females showed larger aortic root dimensions than males, and aortic root diameters increased with age. These findings could prove useful in establishing a normal range of aortic root parameters and accurately diagnosing aortic root disease.

\section{Funding}

National Key R\&D Program of China (2020YFC20 08100).

\section{Disclosure}

Fang Wang and Bin $\mathrm{Lu}$ are co-correspondence authors. The authors report no conflicts of interest in this work.

\section{References}

1. Quail MA, Nordmeyer J, Schievano S, Reinthaler M, Mullen MJ, Taylor AM. Use of cardiovascular magnetic resonance imaging for TAVR assessment in patients with bioprosthetic aortic valves: comparison with computed tomography. Eur J Radiol. 2012;81:3912-3917. doi:10.1016/j.ejrad.2012.07.014

2. Leipsic J, Gurvitch R, Labounty TM, et al. Multidetector computed tomography in transcatheter aortic valve implantation. JACC Cardiovasc Imaging. 2011;4:416-429.

3. Jabbour A, Ismail TF, Moat N, et al. Multimodality imaging in transcatheter aortic valve implantation and post-procedural aortic regurgitation: comparison among cardiovascular magnetic resonance, cardiac computed tomography, and echocardiography. J Am Coll Cardiol. 2011;58:2165-2173. doi:10.1016/j.jacc.2011.09.010
4. Smid M, Ferda J, Baxa J, et al. Aortic annulus and ascending aorta: comparison of preoperative and periooperative measurement in patients with aortic stenosis. Eur J Radiol. 2010;74:152-155. doi:10.1016/j.ejrad.2009.01.028

5. de Heer LM, Budde RP, Mali WP, de Vos AM, van Herwerden LA, Kluin J. Aortic root dimension changes during systole and diastole: evaluation with ECG-gated multidetector row computed tomography. Int J Cardiovasc Imaging. 2011;27:1195-1204. doi:10.1007/s10554011-9838-x

6. Tops LF, Wood DA, Delgado V, et al. Noninvasive evaluation of the aortic root with multislice computed tomography implications for transcatheter aortic valve replacement. JACC Cardiovasc Imaging. 2008;1:321-330. doi:10.1016/j.jcmg.2007.12.006

7. Wood DA, Tops LF, Mayo JR, et al. Role of multislice computed tomography in transcatheter aortic valve replacement. Am J Cardiol. 2009;103:1295-1301. doi:10.1016/j.amjcard.2009.01.034

8. Jilaihawi H, Kashif M, Fontana G, et al. Cross-sectional computed tomographic assessment improves accuracy of aortic annular sizing for transcatheter aortic valve replacement and reduces the incidence of paravalvular aortic regurgitation. $J \mathrm{Am}$ Coll Cardiol. 2012;59:1275-1286. doi:10.1016/j.jacc.2011.11.045

9. Litmanovich DE, Ghersin E, Burke DA, Popma J, Shahrzad M, Bankier AA. Imaging in Transcatheter Aortic Valve Replacement (TAVR): role of the radiologist. Insights Imaging. 2014;5:123-145.

10. Bedogni F, Latib A, De Marco F, et al. Interplay between mitral regurgitation and transcatheter aortic valve replacement with the CoreValve Revalving System: a multicenter registry. Circulation. 2013;128:2145-2153.

11. Lehmkuhl L, Foldyna B, Von Aspern K, et al. Inter-individual variance and cardiac cycle dependency of aortic root dimensions and shape as assessed by ECG-gated multi-slice computed tomography in patients with severe aortic stenosis prior to transcatheter aortic valve implantation: is it crucial for correct sizing? Int J Cardiovasc Imaging. 2013;29:693-703. doi:10.1007/s10554-012-0123-4

12. Perk J, De Backer G, Gohlke H, et al. European Association for Cardiovascular Prevention \& Rehabilitation (EACPR); ESC Committee for Practice Guidelines (CPG). European Guidelines on cardiovascular disease prevention in clinical practice (version 2012). The Fifth Joint Task Force of the European Society of Cardiology and Other Societies on Cardiovascular Disease Prevention in Clinical Practice (constituted by representatives of nine societies and by invited experts). Eur Heart J. 2012;33(13):1635-1701. doi:10.1093/ eurheartj/ehs092

13. Stevenson PH. Height-weight-surface formula for the estimation of surface area in Chinese subjects. Chin J Physiol. 1937;3:327-330.

14. Agmon Y, Khandheria BK, Meissner I, et al. Is aortic dilatation an atherosclerosis-related process? Clinical, laboratory, and transesophageal echocardiographic correlates of thoracic aortic dimensions in the population with implications for thoracic aortic aneurysm formation. $J$ Am Coll Cardiol. 2003;42:1076-1083. doi:10.1016/S07351097(03)00922-7

15. Vahanian A, Himbert D, Brochet E, Depoix JP, Iung B, Nataf P. Transcatheter aortic valve implantation: our vision of the future. Arch Cardiovasc Dis. 2012;105:181-186. doi:10.1016/j.acvd.2012.01.004

16. Wolak A, Gransar H, Thomson LE, et al. Aortic size assessment by noncontrast cardiac computed tomography: normal limits by age, gender, and body surface area. JACC Cardiovasc Imaging. 2008;1:200-209. doi:10.1016/j.jcmg.2007.11.005

17. Davis AE, Lewandowski AJ, Holloway CJ, et al. Observational study of regional aortic size referenced to body size: production of a cardiovascular magnetic resonance nomogram. J Cardiovasc Magn Reson. 2014;16:9. doi:10.1186/1532-429X-16-9

18. Lam CS, Xanthakis V, Sullivan LM, et al. Aortic root remodeling over the adult life course: longitudinal data from the Framingham Heart Study. Circulation. 2010;122:884-890. doi:10.1161/ CIRCULATIONAHA. 110.937839 
19. Schlatmann TJ, Becker AE. Histologic changes in the normal aging aorta: implications for dissecting aortic aneurysm. Am J Cardiol. 1977;39:13-20. doi:10.1016/S0002-9149(77)80004-0

21. Daimon M, Watanabe H, Abe Y, et al. Normal values of echocardiographic parameters in relation to age in a healthy Japanese population: the JAMP study. Circ J. 2008;72:1859-1866.

22. Biaggi P, Matthews F, Braun J, Rousson V, Kaufmann PA, Jenni R. Gender, age, and body surface area are the major determinants of ascending aorta dimensions in subjects with apparently normal echocardiograms. $J$ Am Soc Echocardiogr. 2009;22:720-725. doi:10.1016/j.echo.2009.03.012
23. Hager A, Kaemmerer H, Rapp-Bernhardt U, et al. Diameters of the thoracic aorta throughout life as measured with helical computed tomography. $J$ Thorac Cardiovasc Surg. 2002;123:1060-1066. doi: $10.1067 / \mathrm{mtc} .2002 .122310$

24. Pham MHC, Ballegaard C, de Knegt MC, et al. Normal values of aortic dimensions assessed by multidetector computed tomography in the Copenhagen General Population Study. Eur Heart J Cardiovasc Imaging. 2019;20(8):939-948. doi:10.1093/ehjci/jez012

\section{Publish your work in this journal}

The International Journal of General Medicine is an international, peer-reviewed open-access journal that focuses on general and internal medicine, pathogenesis, epidemiology, diagnosis, monitoring and treatment protocols. The journal is characterized by the rapid reporting of reviews, original research and clinical studies across all disease areas. The manuscript management system is completely online and includes a very quick and fair peer-review system, which is all easy to use. Visit http://www.dovepress.com/ testimonials.php to read real quotes from published authors.

Submit your manuscript here: https://www.dovepress.com/international-journal-of-general-medicine-journal 